\title{
THE RELATIVE CHANGES IN AFFERENT AND EFFERENT ARTERIOLAR RESISTANCE IN THE NORMAL HUMAN KIDNEY
}

\author{
Bx HAROLD LAMPORT
}

(From the Department of Neurology, College of Physicians and Surgeons, Columbia University and The Neurological Institule, New York City)

(Received for publication May 21, 1941)

Recently, the problem of renal physiology has been considerably elucidated by an analysis of the pressure relations existing in the glomerulus. Homer Smith and his collaborators (1) have made certain simplifying assumptions, some of them tacitly, in reaching their conclusions. The validity of these assumptions has been examined and other formulae have been developed (2). The maximum difference between the two formulae for afferent arteriolar resistance occurs at minimum kidney blood flow and is large enough so that, where the relationship between afferent and efferent arteriolar resistance is in question, the formulae developed elsewhere will be employed. It should be understood, however, that the same conclusion has been reached using the equations derived by Smith and his coworkers.

These observers note that the diodrast clearance gives the approximate rate of flow of plasma through the kidney and that blood flow is given by multiplying the diodrast clearance by a constant factor dependent on the hematocrit (1). This factor can be further modified to include another, which corrects for a failure of the nephrons to extract all the diodrast from plasma, and for urinary diodrast derived from red blood cells. In man, this correction is less than $\mathbf{1 0}$ per cent and will be considered part of the hematocrit correction (1).

Smith and his associates noted that "during marked changes in renal blood flow (adrenalin ischemia and pyrogenic hyperemia) the rate of glomerular filtration typically remains unchanged" (1). They then conclude that the constancy of the inulin clearance (filtration rate) is due solely "to the physiological circumstance that changes in renal blood flow are mediated through changes in the tonus of the efferent, rather than the afferent, glomerular arterioles." This is considered to be a consequence of their finding that the effective glomerular blood pressure varies inversely as the blood flow through the efferent arterioles (1).

In Figure 1 is shown Smith et al's data for the relationship in normal kidneys between inulin and diodrast clearances per unit diodrast tubular mass. The solid line marked $M$ is considered by these workers to represent the condition of fixed afferent arteriolar resistance and fixed resistance distal to the efferent arterioles, while the dashed line passing through the origin is considered to represent the condition of fixed efferent arteriolar resistance. The resistances are fixed at the mean basal values. The fact that the observations fall within twice the standard deviation from the line $M$, which passes through the mean basal or "standard" point, is considered evidence that, actually, afferent arteriolar resistance is fixed in the conditions studied. We find, however, that when we let afferent arteriolar resistance be constant in our formula, we obtain the hyperbola $A$, drawn as a solid line. The curve for fixed efferent arteriolar resistance is the much flatter curve $E$, shown by the dot-dash line. It is clear that neither of these curves represents the data in Figure 1, so that even when the rate of glomerular filtration is fixed, both afferent and efferent arteriolar resistances vary considerably.

In our formulae, 1 let:

$$
\begin{aligned}
& H= \frac{\text { whole blood volume }}{\text { plasma volume }}=\frac{1}{1-\text { hematocrit }} \\
& \text { tor also may include any correction for blood flow } \\
& \text { that might be required.) }
\end{aligned}
$$




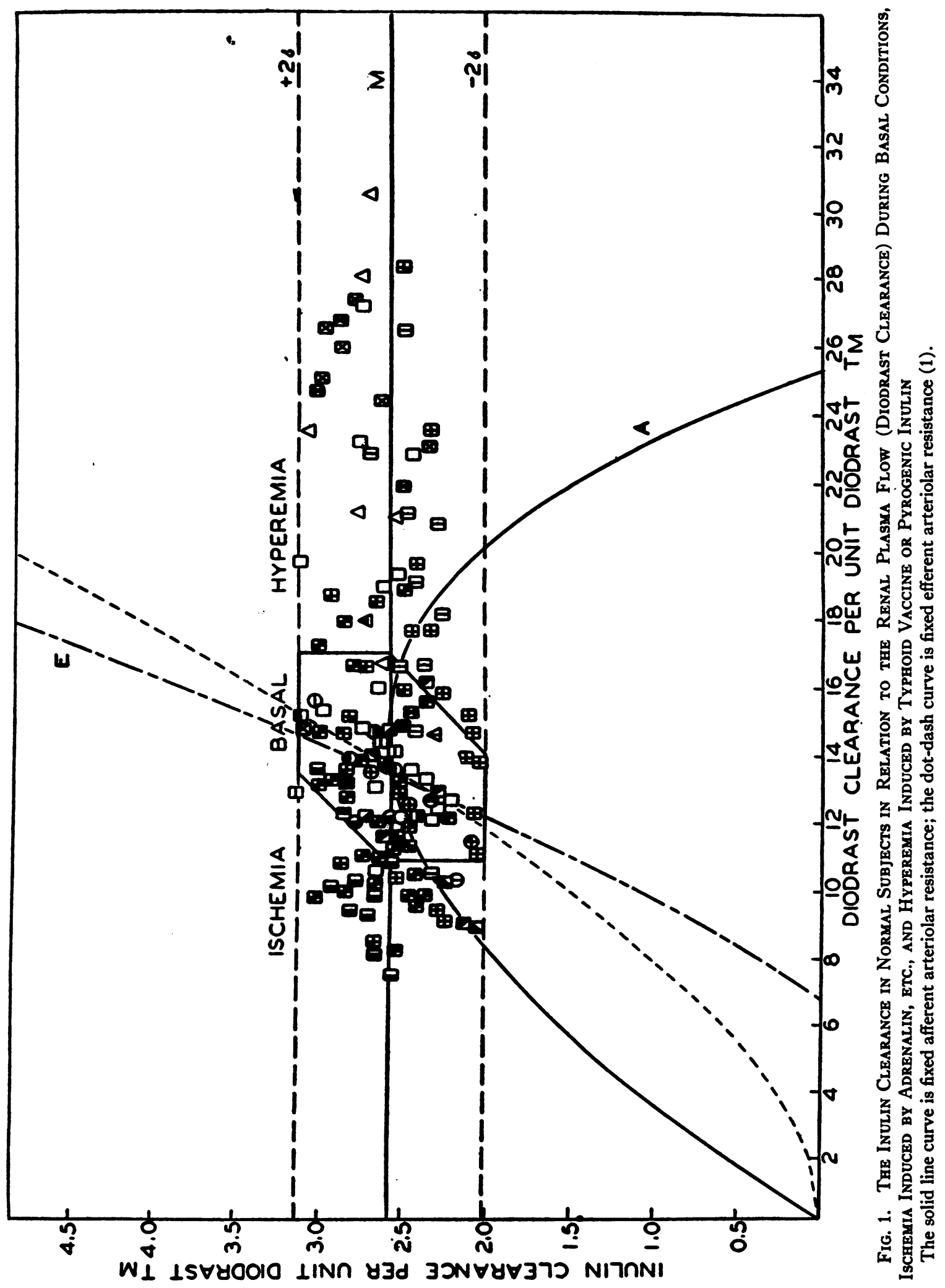


$P_{0}=$ osmotic pressure of unconcentrated blood in $\mathrm{mm}$. $\mathrm{Hg}$.

$P_{0^{\prime}}=$ osmotic pressure of blood concentrated in glomerulus in $\mathbf{~ m m}$. $\mathrm{Hg}$.

$P_{M}=$ average of systolic and diastolic brachial blood pressures in $\mathrm{mm}$. of $\mathrm{Hg}$.

$S=$ concentration of protein in grams per $100 \mathrm{cc}$. of normal human serum.

$R_{\boldsymbol{A}}=$ resistance of afferent arterioles (defined more precisely elsewhere) (2).

$R_{E}=$ resistance of efferent arterioles (2).

$k=$ constant, dependent on hematocrit, equal to 0.47 when hematocrit is $\mathbf{0 . 4 3}$.

As previously shown (2):

$$
\begin{aligned}
R_{A} & =\frac{P_{M}-P_{O^{\prime}}-40}{H D} ; \\
R_{E} & =\frac{(1-k F)\left(P_{O^{\prime}}-P_{O}+10\right)}{H D} ; \\
P_{O^{\prime}} & =\frac{2.34 S}{1-0.0542 S-F} .
\end{aligned}
$$

If we take $S=7.0 ; H=1.755$ (corresponds to hematocrit of 0.43 ); $P_{o}=26.4 ; k=0.47$; and the mean basal condition of $D=13.4$ and $I$ $=2.56$, as was previously done in a clinical application (2); and let $P_{M}=90$, as have Smith and his collaborators, ${ }^{2}$ we have:

$$
D=52.7-\frac{17.3}{0.63-F},
$$

where $R_{A}$ is constant at 0.540 , the mean basal value found for $D=13.4, I=2.56$. And, where $R_{E}$ is fixed at $\mathbf{0 . 8 0 9}$, for the same values of $D$ and $I$, the equation determining the resulting relationship of $D$ and $I$ is:

$$
D=11.55(1-0.47 F)\left(\frac{1}{0.63-F}-1\right) .
$$

While Figure 1 illustrates the fact that neither set of arterioles is fixed when renal blood flow varies, another way of demonstrating this is to compute, from the formulae, the effect on resistance of a 1 per cent decrease in renal blood flow or diodrast clearance, to which it is proportional, while the inulin clearance (glomerular filtration rate) stays constant. We find that such a change in flow at the mean basal point (1) requires approximately a $\mathbf{0 . 2}$ per cent decrease in afferent

\footnotetext{
2 An examination of the papers referred to shows that mean blood pressure does not change much during maximal renal ischemia due to adrenalin.
}

arteriolar resistance and a 0.9 per cent increase in efferent arteriolar resistance.

Smith and his collaborators demonstrate that the consequence of assuming the fall in pressure in the efferent arterioles $\left(P_{E}\right)$ to be inversely proportional to the flow through them $(Q)$ is constancy of glomerular filtration rate, if their formulae are applicable (1). They consider that this inverse relationship is the result of fixed afferent arteriolar resistance. While we have offered evidence that this is not the case, let us determine the meaning of this relationship. In algebraic form, where $W$ is a constant, it becomes:

$$
P_{E}=W / Q ; \quad \text { or } \quad W=P_{E} Q .
$$

This is the analogue of power loss in the form of heat in electricity: watts $=$ volts $X$ amperes. The assumption made, therefore, is really that the efferent arterioles vary in caliber so that the kinetic energy of the flowing blood dissipated in them as heat (friction loss) remains constant. The change in blood viscosity as a result of glomerular hemoconcentration must be considered negligible and osmotic pressure must be considered proportional to hemoconcentration for this relationship to be true (1).

Is it approximately true? If $W$, above, evaluated with regard to the effects of viscosity and non-linear osmotic pressure changes, remains fairly invariant, with changes in renal blood flow, the constancy of efferent arteriolar heat loss could be a good approximation. For $Q$, we use the flow modified to include the effects of viscosity, as justified elsewhere (2), and the formula for $P_{O^{\prime}}$ has been given. We then have:

$$
W=\left(P_{o^{\prime}}-P_{o}+10\right)\left(\frac{H D}{1-k F}\right) \text {. }
$$

Using the normal values, as before:

$$
W=\left(P_{o^{\prime}}-16.4\right) \frac{1.755 D}{1-0.47 F} \text {. }
$$

Evaluating $W$ at two different ranges of blood flow, ischemia and hyperemia, by letting $I$ be constant at its mean value of $2.56 \mathrm{cc}$. and letting $D=7$ and $20 \mathrm{cc}$. per minute per unit diodrast tubular mass, we determine the change in $W$ produced by a 1 per cent decrease in $D$ (1 per cent 
decrease in renal blood flow). We find that when $D=7$, a 1 per cent decrease in $D$ causes a 1.2 per cent increase in $W$, which is 442; when $D=20$, a 1 per cent decrease in $D$ produces an increase in $W$ of 0.6 per cent from 258. It is therefore clear that the "constant" $W$ varies considerably and the friction heat loss in the efferent arterioles is not approximately fixed.

\section{SUMMARY}

1. Formulae for the afferent and efferent arteriolar resistance to renal blood flow in man have been applied to available data for normal subjects under ischemic, basal, and hyperemic renal conditions.

2. It has been found that the resistance of both sets of arterioles vary simultaneously in maintaining constant glomerular filtration in the basal state and in adrenalin ischemia and pyrogenic hyperemia of the human kidney.
3. At the mean basal state, a 1 per cent decrease in renal blood flow per unit diodrast tubular mass is caused by approximately a 0.2 per cent decrease in afferent arteriolar resistance and a 0.9 per cent increase in efferent arteriolar resistance.

4. The heat loss due to friction of the blood flowing through the efferent arterioles is not approximately constant.

\section{BIBLIOGRAPHY}

1. Smith, H. W., and others, Glomerular dynamics in the normal human kidney. J. Clin. Invest., 1940, 19, 751.

2. Lamport, H., Formulae for afferent and efferent arteriolar resistance in the human kidney: an application to the effects of spinal anesthesia. J. Clin. Invest., 1941, 20, 535.

3. Goldring, W., and others, Relations of effective renal blood flow and glomerular filtration to tubular excretory mass in normal man. J. Clin. Invest., 1940, 19, 739. 